\title{
Tracking and Characterization of Fragments in a Beating Heart Using 3D Ultrasound for Interventional Guidance
}

\author{
Paul Thienphrapa ${ }^{1,2}$, Haytham Elhawary ${ }^{1}$, Bharat Ramachandran ${ }^{1}$, \\ Douglas Stanton ${ }^{1}$, and Aleksandra Popovic ${ }^{1}$ \\ 1 Philips Research North America, Briarcliff Manor, NY, USA \\ aleksandra.popovic@philips.com \\ 2 ERC CISST/LCSR, Johns Hopkins University, Baltimore, MD, USA
}

\begin{abstract}
Fragments generated by explosions and similar incidents can become trapped in a patient's heart chambers, potentially causing disruption of cardiac function. The conventional approach to removing such foreign bodies is through open heart surgery, which comes with high perioperative risk and long recovery times. We thus advocate a minimally invasive surgical approach through the use of 3D transesophageal echocardiography (TEE) and a flexible robotic end effector. In a phantom study, we use 3D TEE to track a foreign body in a beating heart, and propose a modified normalized cross-correlation method for improved accuracy and robustness of the tracking, with mean RMS errors of $2.3 \mathrm{~mm}$. Motion analysis of the foreign body trajectory indicates very high speeds and accelerations, which render unfeasible a robotic retrieval method based on following the tracked trajectory. Instead, a probability map of the locus of the foreign body shows that the fragment tends to occupy only a small sub-volume of the ventricle, suggesting a retrieval strategy based on moving the robot end effector to the position with the highest spatial probability in order to maximize the possibility of capture.
\end{abstract}

\section{Introduction}

Penetration of a fragment into the heart is a common injury in both civilian accidents and military warfare [9], 11. Small caliber bullets and small shell fragments with low velocity tend to circulate freely in the chambers, potentially leading to arrhythmia, occlusion, and possibly death [3], 8]. The most frequent treatment approach to removing these fragments involves open surgery via a median sternotomy and cardiopulmonary bypasss (CPB) [9], a highly invasive procedure requiring a long recovery period. Risks of this procedure include bacterial mediastinitis, hemolysis, blood clotting, and air embolism.

The long term objective of this work is to develop a flexible robotic system for minimally invasive retrieval of fragments from a beating heart. In the envisioned clinical scenario, the end effector is inserted transapically into the heart (Fig. 1 [left]) after selection of the fragment via preoperative imaging. Then, under ultrasound guidance, the robot moves to capture the fragment; distal dexterity is 
needed to adequately cover the workspace. A minimally invasive approach can significantly improve the management of cardiac fragments by reducing risk and mortality, improving postoperative recovery, and potentially reducing operating room times. The objectives of the present study are (1) to demonstrate the utility of 3D transesophageal echocardiography (TEE) in tracking a target moving rigorously against a dynamic background; (2) to develop real-time tracking of the fragments; and (3) to analyze its motion in the context of a robotic system.

The use of $3 \mathrm{D}$ ultrasound for interventional systems is a fairly recent development due to high cost, low resolution, and inability to access real-time data streams [2]. In 7], markers were attached to an instrument to allow for imagebased tracking. Variability in the readings was less than $1 \mathrm{~mm}$. The instrument was subsequently driven towards targets in a water tank, reaching them with an error of $1.2 \mathrm{~mm} \mathrm{4}$. Experiments with low-resolution 3D catheter transducers [10] yielded errors of around $3 \mathrm{~mm}$. Targets were static in these experiments.

Ferrous shrapnel can be difficult to detect in ultrasound when adjacent to other hyperechoic surfaces. In [5, a variable magnetic field is used to induce vibrations in shrapnel, thus illuminating it under color flow Doppler and allowing it to be located within an error of $1.06 \mathrm{~mm}$.

The performance requirements for tracking a foreign body in the heart are more akin to the 1-degree-of-freedom (DOF) heartbeat compensation device of 12. Predictive control was employed to track heart structures in one dimension with positive results. On the other hand, cardiac motions are more predictable than that of an object moving freely within the heart. Due to a variety of influences, foreign bodies are prone to seemingly arbitrary motions. A predictive control scheme is less likely to be effective in this case. The main contribution of this paper is the tracking of an unpredictably moving target using 3D TEE, for the purpose of developing a retrieval strategy by a flexible robotic device inserted through the apex of the heart.

\section{Experimental Setup}

A system for studying the problem of minimally invasive evacuation of fragments from a beating heart is illustrated in Fig. 1 (center). It includes an ultrasound system, a beating heart phantom, and a workstation $(2.3 \mathrm{GHz}$ Intel Xeon, $4 \mathrm{~GB}$ of RAM) that acquires live streaming ultrasound volumes over TCP/IP.

The Philips iE33 xMATRIX Echocardiography System is used with the X7-2t 3 D TEE probe. Each image has a resolution of $128 \times 48 \times 112$ voxels of size 0.81 , 0.96 , and $0.98 \mathrm{~mm}$, spanning a field of view of $60^{\circ}$ azimuth, $30^{\circ}$ elevation, and $12 \mathrm{~cm}$ depth respectively. Gain and compression are set at $47 \%$ and $40 \mathrm{~dB}$.

The beating heart phantom is a custom developed multi-modality phantom compatible with X-ray, ultrasound, and MR imaging. Made of polyvinyl acetate (PVA), it is a full replica of a human heart and resides in an acrylic glass water tank. Two servo-actuated pneumatic pistons pump water into and out of the phantom to create the deformable effect of a heartbeat and blood flow. Each piston pumps about $18 \mathrm{ml}$ of water per heartbeat; its motion is given in Fig. 1 


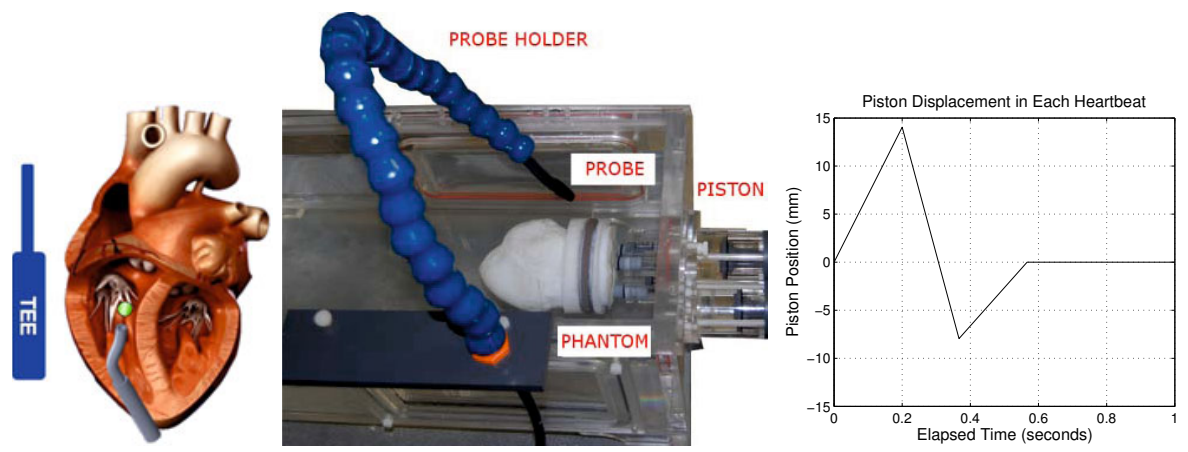

Fig. 1. (Left) Robotic fragment retrieval from the heart under 3D TEE guidance. (Center) Arrangement of the TEE probe and the heart phantom. (Right) Piston displacement in the beating heart phantom for one heartbeat.

(right). An experimental heart rate of $1 \mathrm{~Hz}$ and stroke volume lower than in healthy humans were chosen to mimic surgical [6] and post-injury cardiovascular conditions.

As an early approximation, a $3.2 \mathrm{~mm}$ steel ball was selected to act as a fragment due to its likeness to the clinical case in terms of size (typically 2-5 mm) and material. The fragment was imaged under 3D TEE at 20 frames per second (fps) and manually segmentated from five datasets $(n=5)$ of 400 frames $(20$ seconds) each. This duration was empirically found to capture most of the activity that would occur in a given trial. Furthermore, 20 heart cycles permit robust testing of online tracking methods - a previous study reports on an in vivo instrument tracking experiment using a five-second window 44. Fig. 2 shows the fragment in the heart phantom manually outlined in the 3D TEE image.
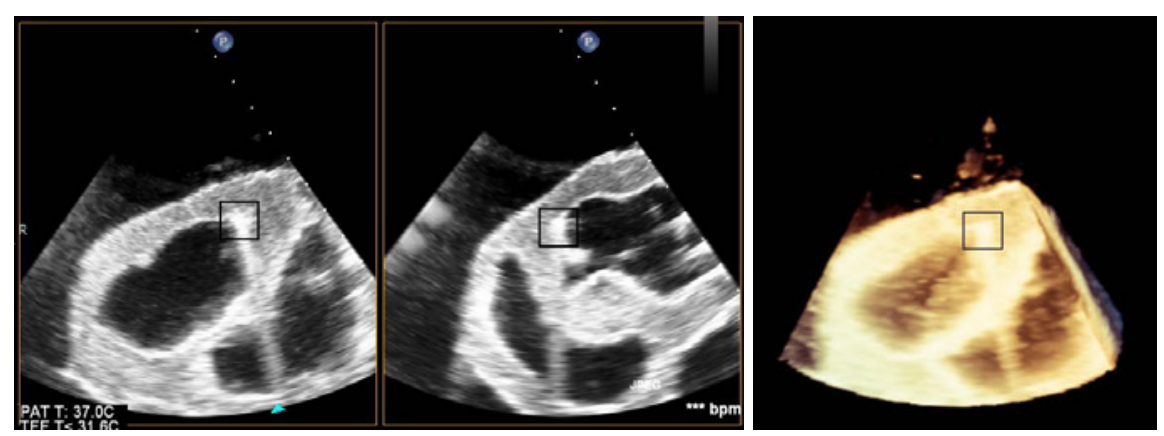

Fig. 2. (Left and center) Orthogonal slices showing the fragment (outlined) in the right ventricle of the heart phantom at rest. (Right) A three-dimensional view. 


\section{Real-Time Tracking of the Fragment}

\subsection{Approach}

Normalized cross-correlation (NCC) is a well-known image processing algorithm used for template matching. The correlation coefficient at a voxel, $R(u, v, w)$, for a template $t(x, y, z)$ is given in (1). Normalization, accomplished by subtracting the mean from both the template and the image under it $(\bar{t}$ and $\bar{f}$ respectively) and dividing by their standard deviations, provides for robustness against changes in intensity, which can occur as both the fragment and background move.

$$
R(u, v, w)=\frac{\sum_{x, y, z}[f(x, y, z)-\bar{f}][t(x-u, y-v, z-w)-\bar{t}]}{\sqrt{\sum_{x, y, z}[f(x, y, z)-\bar{f}]^{2} \sum_{x, y, z}[t(x-u, y-v, z-w)-\bar{t}]^{2}}}
$$

The fragment was selected interactively from the first image frame to define the template for the algorithm to track in subsequent frames. A template of size $10 \times 10 \times 10$ voxels was suitable for enclosing the entire fragment, while a search space of $40 \times 30 \times 30$ was sufficient to capture displacements between frames. The low computation time of NCC (38 ms per frame) makes it especially suitable for tracking when real-time performance is required.

The coefficient $R$ in (11) is a real number in $[-1,1]$, with higher values indicating a close match and values tending toward -1 signifying a match of opposite intensity. The location of the maximum $R\left(R_{\max }\right)$ found in a search space is taken to be the position of the fragment. However, a weakness of NCC is that it relies on consistency in the apperance of the target, whereas apperances may

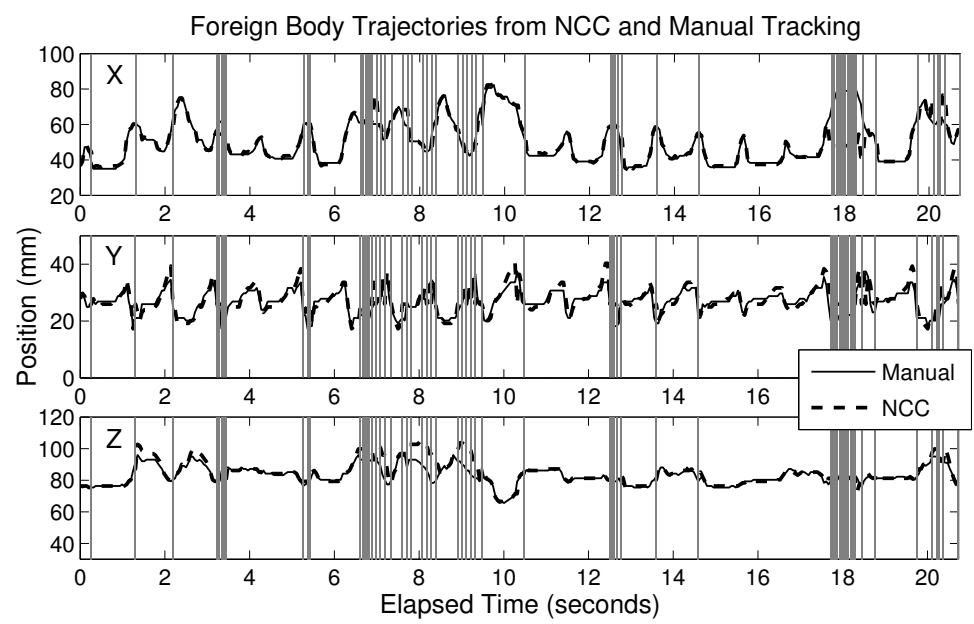

Fig. 3. Fragment trajectories based on NCC vs. manual tracking. Dropped frames are marked by vertical lines. 
vary in ultrasound due to noise, artifacts, and low resolution. This can lead to the target being incorrectly identified in some frames. A low $R_{\max }$ suggests that NCC was not confident in its decision, and errant tracking can be avoided by ignoring such frames. In our experiments, a frame $i$ is dropped if $R_{i, \max }$ is less than $c$ standard deviations below the mean of the dataset: $R_{i, \max }<R_{\text {mean }}-c \cdot R_{s d}$. The value of $c$ can be tuned to the level of confidence desired; we found $c=0.9$ to exhibit a reasonable compromise between spatial and temporal accuracy.

\subsection{Results}

Accuracy. Fig. 3 shows a trace of the fragment as determined by NCC, with manually-segmented fragment localization serving as a basis for evaluation 1 Table 1 lists the number of dropped frames and accuracy for all datasets. NCCbased fragment tracking with detection of low confidence intervals (post-drop error) was accurate to within $2.3 \mathrm{~mm}$ rms after dropping $18.5 \%$ of frames. As expected, in the case of NCC without frame dropping (pre-drop error), tracking was lost in some frames resulting in mean errors that were almost twice as high $(4.3 \mathrm{~mm})$. For frames in which the reported fragment location is unavailable or doubtful, it is preferable to have the system wait until tracking is recovered, allowing the robotic retrieval instrument to stay in its current position or to be placed at a safe location if the uncertainty is prolonged over several frames.

Table 1. Accuracy of NCC referenced to data extracted manually

\begin{tabular}{|l||c|c|c|c|c||c|c|c|c|}
\hline \multicolumn{1}{|c||}{} & \multicolumn{4}{c||}{ Datasets } & \multicolumn{4}{c|}{ Aggregate } \\
\hline \multicolumn{1}{|c||}{ Parameter } & 1 & 2 & 3 & 4 & 5 & Min & Mean & Max & SD \\
\hline Pre-Drop Error $(\mathrm{mm} \mathrm{rms)}$ & 6.2 & 4.8 & 5.3 & 2.5 & 2.6 & 2.5 & 4.3 & 6.2 & 1.7 \\
\hline Post-Drop Error $(\mathrm{mm} \mathrm{rms)})$ & 2.0 & 3.1 & 2.6 & 1.8 & 2.1 & 1.8 & 2.3 & 3.1 & 0.5 \\
\hline$\%$ Frames Dropped & 20.9 & 15.5 & 20.1 & 22.9 & 13.3 & 13.3 & 18.5 & 22.9 & 4.0 \\
\hline $100 \times R_{\text {mean }}$ & 80.4 & 71.0 & 83.6 & 85.1 & 84.0 & 71.0 & 80.8 & 85.1 & 5.8 \\
\hline $100 \times R_{s d}$ & 9.2 & 8.5 & 10.3 & 4.8 & 3.7 & 3.7 & 7.3 & 10.3 & 2.9 \\
\hline
\end{tabular}

Table 2. Fragment motion parameters obtained from 3D TEE images

\begin{tabular}{|c||c|c|c|c|c||c|c|c|c|}
\hline \multicolumn{1}{|c||}{} & \multicolumn{4}{c||}{ Datasets } & \multicolumn{4}{c|}{ Aggregate } \\
\hline Parameter & 1 & 2 & 3 & 4 & 5 & Min & Mean & Max & SD \\
\hline Range $(\mathrm{mm})$ & 51.4 & 49.0 & 47.5 & 23.5 & 29.8 & 23.5 & 40.2 & 51.4 & 12.7 \\
\hline Speed $(\mathrm{mm} / \mathrm{s})$ & 336.2 & 334.2 & 530.0 & 208.7 & 308.3 & 208.7 & 343.5 & 530.0 & 116.5 \\
\hline Accel. $\left(\mathrm{m} / \mathrm{s}^{2}\right)$ & 5.3 & 9.5 & 9.1 & 6.2 & 8.7 & 5.3 & 7.8 & 9.5 & 1.9 \\
\hline
\end{tabular}

Motion Characterization. Table 2 summarizes the extracted motion parameters. The average range of motion is $40.2 \mathrm{~mm}$ over three axes. While there is some correspondence between fragment motion and heartbeats (see Fig. 3), there is an element of motion that can seem arbitrary. Frequency domain analysis (Fig. 4) supports this observation with the primary peak located at $1 \mathrm{~Hz}$,

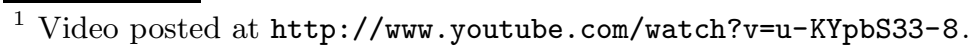



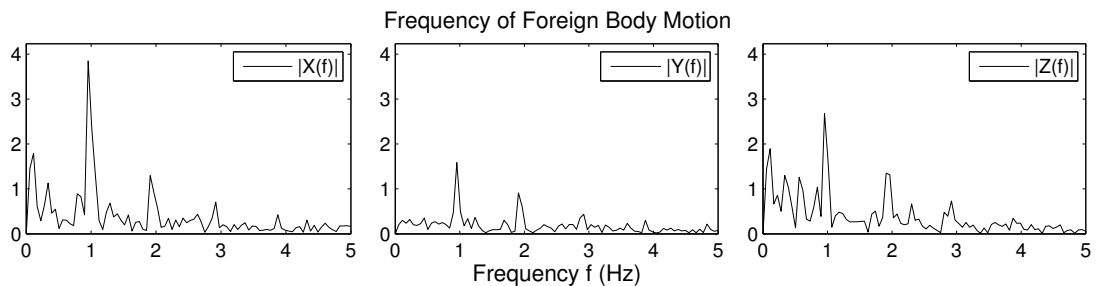

Fig. 4. Frequency components of fragment motion

but with spectral power at both lower (drift) and higher frequencies (heartbeat harmonics) suggesting a more complex behavior. Previous studies have found higher frequency components in the mitral valve as well [12].

\section{Probability Map}

The fragment reaches speeds of about $343.5 \mathrm{~mm} / \mathrm{s}$. Similar speeds have been reported for the heart wall $(300 \mathrm{~mm} / \mathrm{s}$ [1] $)$ and mitral valve $(200 \mathrm{~mm} / \mathrm{s} \mathrm{[12]})$. Acceleration $\left(7.8 \mathrm{~m} / \mathrm{s}^{2}\right.$ on average) is higher than figures reported for the mitral valve $\left(3.8 \mathrm{~m} / \mathrm{s}^{2}[12]\right)$. A retrieval strategy based on following the trajectory of the foreign body using a robotic end effector would be extremely challenging given these high speeds and accelerations. Furthermore, the uncertainties inherent in a beating heart environment may make it hazardous to do so. We thus propose a different approach for retrieval of the foreign body, wherein the real-time tracking method described in this paper is used to build a spatial probability map of the fragment, based on its past locations. The map can then be used to position a dexterous robotic device in the most visited locations of the fragment in order to maximize the possibility of capture.
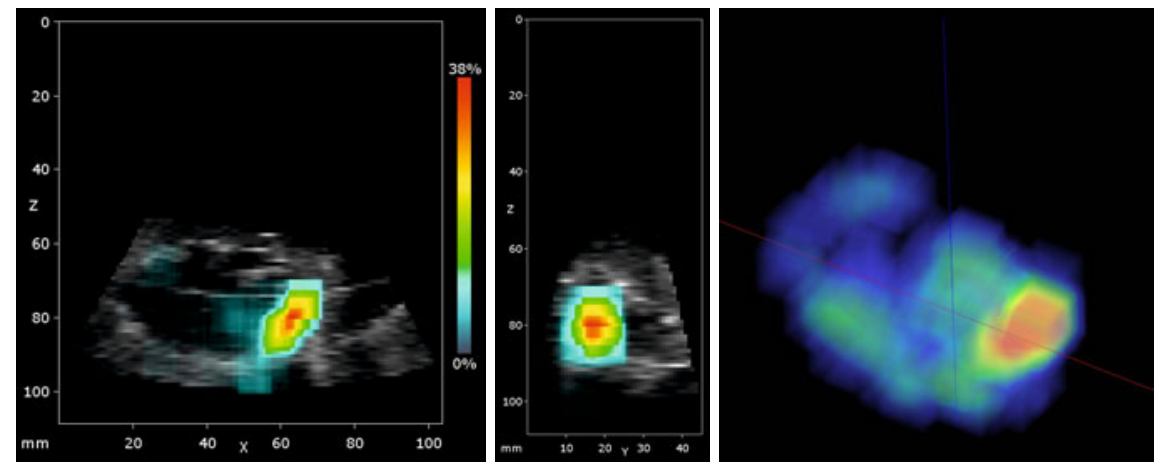

Fig. 5. Probability map of the fragment position. (Left and center) Coronal and sagittal slices of the map. (Right) Three-dimensional view. 


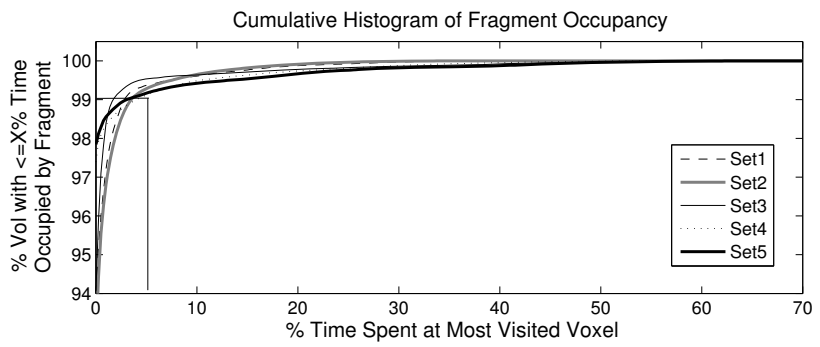

Fig. 6. Cumulative histograms showing how frequently voxels are visited by the fragment. Around $\mathrm{Y}=94 \%$ of the volume is never touched $(\mathrm{X}=0 \%)$, and roughly $\mathrm{Y}=99 \%$ of the volume is occupied at most $\mathrm{X}=5 \%$ of the time.

Table 3. Tendency of fragment to visit certain locations. The first row describes the percentage of the volume occupied at least $25 \%$ of the time. The second lists the maximum percentage of time that any one voxel was occupied.

\begin{tabular}{|c||c|c|c|c|c||c|c|c|c|}
\hline \multicolumn{1}{|c||}{} & \multicolumn{4}{c||}{ Datasets } & \multicolumn{4}{c|}{ Aggregate } \\
\hline Parameter & 1 & 2 & 3 & 4 & 5 & Min & Mean & Max & SD \\
\hline 25\% FB Occupancy & 0.07 & 0.03 & 0.17 & 0.19 & 0.24 & 0.03 & 0.14 & 0.24 & 0.09 \\
\hline Max Occupancy Level & 39.8 & 38.0 & 57.5 & 54.0 & 63.0 & 38.0 & 50.5 & 63.0 & 11.1 \\
\hline
\end{tabular}

A qualitative representation of the fragment motion, generated using the tracking approach described above, is shown in Fig. 5. Warmer (red) regions indicate where the fragment is more likely to be located based on fragment traces. The observed tendency of the fragment to be localized in a subsection of the overall ventrical volume suggests the viability of a more relaxed retrieval approach based on aiming the dexterous robotic end effector at the volume of highest probability of fragment location. Histograms (Fig. 6] and Table 3) show that on the average across datasets, the fragment spends up to $50.5 \%$ of the time in the most visited voxel. Although it is shown in the frequency domain (Fig. 4) that the motion of the fragment is more irregular than the motion of the heart wall, predictive models using the spatial position of the fragment can be used to move the robot to a 'waiting' position, i.e. a position of high probability.

\section{Conclusions and Future Work}

In this paper, the motion of a fragment in a beating heart phantom is quantified using motion tracking in 3D TEE images. We show that the fragment motion is fast, abrupt, and often unpredictable. This provides valuable insight regarding the design of an interventional system that is capable of catching the particle. The next milestones will involve the design of a robot control scheme that uses the spatial probability map to safely retrieve the fragment, as well as studies of different fragment counts and physical properties. 


\section{References}

1. Kostis, J.B., Mavrogeorgis, E., Slater, A., Bellet, S.: Use of a range-gated, pulsed ultrasonic Doppler technique for continuous measurement of velocity of the posterior heart wall. Chest 62(5), 597 (1972)

2. Krupa, A., Fichtinger, G., Hager, G.D.: Real-time motion stabilization with Bmode ultrasound using image speckle information and visual servoing. Int. J. Robot. Res. 28(10), 1334-1354 (2009)

3. Marshall, A.J., Ring, N.J., Newman, P.L.: An unexplained foreign body in the myocardium. J. Royal Soc. Med. 95, 250-251 (2002)

4. Novotny, P.M., Stoll, J.A., Vasilyev, N.V., del Nido, P.J., Dupont, P.E., Zickler, T.E., Howe, R.D.: GPU-based real-time instrument tracking with threedimensional ultrasound. Med. Image Anal. 11, 458-464 (2007)

5. Rogers, A.J., Light, E.D., Smith, S.W.: 3-D ultrasound guidance of autonomous robot for location of ferrous shrapnel. IEEE Trans. Ultrason., Ferroelectr., Freq. Control 56(7), 1301-1303 (2009)

6. Ryan, K.L., Cooke, W.H., Rickards, C.A., Lurie, K.G., Convertino, V.A.: Breathing through an inspiratory threshold device improves stroke volume during central hypovolemia in humans. J. Appl. Physiol. 104(5), 1402-1409 (2008)

7. Stoll, J.A., Novotny, P.M., Howe, R.D., Dupont, P.E.: Real-time 3D ultrasoundbased servoing of a surgical instrument. In: IEEE Int. Conf. on Robotics and Automation, pp. 613-618 (2006)

8. Symbas, P.N., Picone, A.L., Hatcher, C.R., Vlasis-Hale, S.E.: Cardiac missiles. A review of the literature and personal experience. Ann. Surg. 211(5), 639-647 (1990)

9. United States Department of Defense: Emergency War Surgery: Third United States Revision, s.l., 16: Thoracic Injuries (2004)

10. Whitman, J., Fronheiser, M.P., Smith, S.W.: 3-D ultrasound guidance of surgical robotics using catheter transducers: feasibility study. IEEE Trans. Ultrason., Ferroelectr., Freq. Control 55(5), 1143-1145 (2008)

11. Williams, J.C., Elkington, W.C.: Slow progressing cardiac complications - a case report. J. Chiropr. Med. 7, 28-33 (2008)

12. Yuen, S.G., Kettler, D.T., Novotny, P.M., Plowes, R.D., Howe, R.D.: Robotic motion compensation for beating heart intracardiac surgery. Int. J. Robot. Res. 28(10), 1355-1372 (2009) 